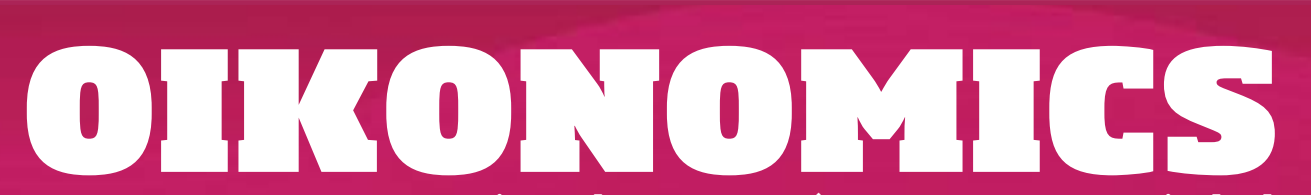

Revista de economía, empresa y sociedad

INNOVACIÓN EN PROCESOS

\title{
Outsourcing de las actividades logísticas. ¿Cómo generamos valor añadido?
}

\section{Oriol Closa Noguera}

Profesor colaborador del máster de Dirección de operaciones y logística integral (UOC)

RESUMEN Las compañías tienden cada vez más hacia la externalización o el outsourcing de todas aquellas actividades que no forman parte de su core business, y en las que no pueden generar valor añadido debido a su falta de especialización o conocimiento específico.

En el momento de la toma de decisiones, las variables que pueden influir más en la externalización de un servicio son el valor que aporta a la actividad de la compañía, la necesidad de hacer variables los costes de gestión o estructurales y la flexibilidad operacional.

Es muy importante analizar el outsourcing desde todas las vertientes posibles, pero sobre todo hay que ser objetivo en la forma de estimar, económica y productivamente, los pros y contras de la decisión. Además, se debe tener en cuenta la estrategia general de la compañía, de forma que todo esté perfectamente alineado y encaje sin fisuras.

Finalmente, conviene ser muy cuidadoso con el tipo de contrato que acordamos con las empresas que ofrecen servicios de externalización logística, puesto que será la base para garantizar el cumplimiento de los objetivos financieros y productivos.

En este artículo nos centraremos en las actividades logísticas que pueden ser externalizables, los motivos de su externalización y el tipo de proveedores de este tipo de servicios. 
PALABRAS ClAVE flexibilidad; especialización; core business; valor añadido; outsourcing; externalización

\section{Outsourcing in logistics activities. How do we generate added value?}

ABSTRACT Companies increasingly tend to outsource any activities that do not form part of their core business, as well as any activities that do not enable them to generate added value, due to their lack of specialization or specific knowledge.

When making decisions in this respect, the variables that may most influence whether a service should be outsourced are the value that the activity generates for the company, the need to make management and structural costs variable, and operational flexibility.

It is essential to analyse outsourcing from all possible angles but, most importantly, we have to be objective when it comes to evaluating the pros and cons of the decision from an economic and production point of view. Moreover, the company's general strategy must be taken into account to ensure that any outsourcing decisions are perfectly aligned to it, without any discrepancy.

Last but not least, we should take great care in terms of the kind of agreement that we reach with the companies offering logistics outsourcing services, as this will be the foundation for ensuring that we meet the financial and production objectives set.

In this article, we focus on logistics activities that may be suitable for outsourcing, the reasons for outsourcing these activities and the type of suppliers of this kind of services.

KEYWORDS flexibility; specialization; core business; added value; outsourcing

\section{Introducción}

Según el Diccionario de empresa de Web Finance, la externalización, o el término anglosajón outsourcing, es «la contratación a terceros de actividades no relevantes para liberar efectivo, personal, tiempo e instalaciones para actividades en las que 
la empresa tenga ventajas competitivas». Por tanto, la subcontratación es «una situación en que una empresa utiliza otra organización para hacer algunos de sus trabajos, en vez de utilizar sus propios empleados para hacerlo».

Uno de los cambios significativos en el negocio global de hoy es la tendencia al outsourcing. La esencia de esta tendencia es subcontratar las actividades a compañías externas especializadas en un determinado ámbito. Esto, por un lado, ofrece a la empresa la oportunidad de proporcionar las tareas de una manera eficaz; y, por otro lado, implica recibir beneficios a partir de negociaciones y en la gestión del pricing. La externalización es especialmente importante en la logística, puesto que incluye muchas operaciones entre la gestión de las materias primas hasta la entrega del producto final. Las empresas tienden a externalizar todo tipo de operaciones logísticas, incluyendo el almacenamiento, la planificación, el transporte de mercancías y la logística inversa.

\section{El proceso de externalización de las actividades logísticas}

En general, todas las operaciones logísticas que se pueden externalizar se pueden dividir en tres categorías:

1. Logística física y entrega: almacenamiento, empaque y flujo físico de mercancías.

2. Logística no física (información): servicios de gestión de operaciones.

3. Logística inversa: retornos, reparaciones y depósito de devoluciones. (RMA: return merchandise authorization).

Cada empresa tiene que decidir cuántas y qué actividades particulares quiere subcontratar. Esta decisión se basa en la distinción entre actividades core (principales) y non-core. Las actividades non-core se determinan a partir de la actividad de la compañía, la volatilidad del mercado y de la especialización de los recursos internos. Principalmente, son aquellas actividades en las que una compañía no aporta valor añadido, al no tratarse de su área de negocio o conocimiento, y, en cambio, generan riesgos tanto en los costes como en los procesos estructurales. Las actividades core son actividades que determinan el éxito competitivo de la empresa y son relevantes porque permiten obtener un alto valor al cliente. Es por este motivo que las actividades non-core tienden a ser externalizadas.

En la tabla 1 podemos observar el resultado del estudio realizado durante más de veinte años sobre el porcentaje de uso del outsourcing en la actividad logística. Se trata de una muestra realizada por Capgemini Consulting en diferentes sectores (farmacia, automoción, moda, alimentación, tecnología, etc.) y con diferentes proveedores de servicios logísticos, actualizada en 2017. 
Tabla 1. Porcentaje de subcontratación de los servicios logísticos 3PL

\begin{tabular}{|c|c|}
\hline Servicios logísticos subcontratados & Porcentaje \\
\hline Transporte nacional & $86 \%$ \\
\hline Almacenamiento & $66 \%$ \\
\hline Transporte internacional & $60 \%$ \\
\hline Transporte de carga & $44 \%$ \\
\hline Corretaje aduanero & $42 \%$ \\
\hline Planificación y gestión del transporte & $36 \%$ \\
\hline Cross-docking & $34 \%$ \\
\hline Auditoría y pago de la factura de la carga & $32 \%$ \\
\hline Gestión de inventarios & $24 \%$ \\
\hline Gestión y cumplimiento de pedidos & $24 \%$ \\
\hline Logística inversa & $23 \%$ \\
\hline Etiquetaje, embalaje, montaje & $22 \%$ \\
\hline $\begin{array}{l}\text { Servicios de consultoría de la cadena de suministro por parte de } \\
\text { los 3PL }\end{array}$ & $19 \%$ \\
\hline Servicios de TI & $17 \%$ \\
\hline Gestión de la flota & $15 \%$ \\
\hline Logística de servicio de piezas & $12 \%$ \\
\hline LLP/4LP & $10 \%$ \\
\hline Servicio al cliente & $9 \%$ \\
\hline
\end{tabular}

Fuente: Langley et al., 2017, pág. 12, tabla 1.

La idea principal de servicios de outsourcing es que no hay que utilizar los recursos propios de la compañía para llevar a cabo las operaciones logísticas. Los proveedores de servicios pueden ofrecer una amplia gama de servicios logísticos a precios asequibles, contribuyendo de forma significativa al desarrollo y aumento de la rentabilidad empresarial, así como a la competitividad de las empresas.

Las actividades que no se deberían subcontratar son las siguientes: 
1. Actividades core que aportan a la empresa el mayor beneficio.

2. Actividades en que la empresa está especializada y tiene una buena base de conocimientos.

3. Funciones con alto impacto por parte del cliente, porque en caso de subcontratación la empresa pierde una parte del control del feedback de los clientes y no tiene posibilidad de reaccionar rápidamente.

4. Actividades que son ineficientes y que actualmente causan dificultades operativas a la empresa. Es mejor resolver inicialmente todos los problemas internamente.

5. Actividades que requieren conocimientos especializados. Por ejemplo, la empresa puede tener una tecnología específica que se creó especialmente para alguna de sus actividades. Además, esta tecnología podría ser propiedad de la empresa.

En cualquier caso, hay algunas preguntas importantes que una empresa tiene que responder una vez se ha tomado la decisión de externalizar. Las principales cuestiones se refieren inicialmente a la estrategia que tiene actualmente la compañía y a los cambios estratégicos que está esperando. Es muy importante combinar y alinear la estrategia de la empresa con la estrategia del proveedor elegido, puesto que socios con diferentes objetivos no pueden tener relaciones a largo plazo.

Antes de iniciar una relación, también es esencial establecer las fronteras en la subcontratación. Después de esto, el paso siguiente importante es elegir el método de evaluación para seleccionar un proveedor y de esta manera evitar riesgos, tanto en el proceso de selección como a posteriori, en el rendimiento de este.

Para estimular y asegurar la asociación, todas las bonificaciones para lograr los objetivos deseables tendrían que ser discutidas con antelación, así como las sanciones por la baja prestación de los servicios contratados. Es imprescindible redactar un contrato vinculante en el que se definan las tarifas por servicio y los $\mathrm{KPl}^{1}$ a partir de los cuales evaluaremos al proveedor. Se ha de elaborar también un plan de contingencia para asegurar la continuidad operacional en cualquier caso y situación.

Los KPI más comunes que se tendrían que incluir en un contrato son:

1. Producción = Unidades u órdenes de distribución movidas por hora.

2. Productividad $=$ Unidades $u$ órdenes de distribución por persona.

3. Costes de distribución y transporte.

4. $\%$ OTD $=$ Porcentaje de unidades entregadas a tiempo.

1. $\mathrm{KPI}=$ key performance indicators. 
5. $\%$ Fiabilidad del inventario $=$ Porcentaje de fiabilidad del inventario .

6. $\%$ Incidencias $=$ Porcentaje de incidencias en la entrega final.

7. \% Capacidad de consolidación de envíos.

La última cuestión a tener en cuenta hace referencia al problema de la conexión entre las organizaciones. No solo en el ámbito de los datos informáticos, sino también en la relación entre la gestión y la administración de ambas partes.

En la tabla 2 vemos un resumen de los riesgos y oportunidades cuando analizamos la subcontratación de los servicios logísticos.

Tabla 2. Riesgos y oportunidades de la subcontratación de servicios logísticos

\section{Oportunidades}

- Reducción de coste. La delegación de algunos procesos a un tercero dará lugar a una disminución significativa de los costes de inventario, transporte, almacenamiento y personal.

- Foco sobre actividades core que son más vitales para una empresa.

- Incremento de la eficiencia, la flexibilidad y la productividad.

- Control de calidad interno.

- Reducción de riesgos, puesto que será el personal altamente cualificado del tercero el responsable de todos los procesos y de todas las instalaciones que proporcionan los servicios contratados

- Uso proporcionado y variable de recursos

- Reducción de la necesidad de utilizar tecnologías propias y acceder utilizando innovaciones.

- Ampliación de los servicios al cliente, expansión del conocimiento del mercado.

\section{Riesgos}

- Los ahorros no realizados con posibilidades de aumentar los costes. En general, la reducción de costes es uno de los motivos principales de la externalización. Sin embargo, no significa que la empresa logre la optimización del control de costes.

- La reducción de puestos de trabajo para los empleados propios puede provocar pérdidas de personal altamente cualificado.

- Contractar o hacer una selección equivocada del partner.

- Problemas con proveedores (rendimiento deficiente o malas relaciones).

- Dependencia de un proveedor o ser cautivos operacionalmente.

- Clientes no satisfechos como resultado del rendimiento incorrecto del proveedor de servicios.

- Acceso por parte del proveedor a datos confidenciales. 


\section{Tipo de proveedores de servicios logísticos}

La externalización logística contiene cinco niveles de los proveedores: del 1PL a 5PL, que se describen a continuación (Waters, 2014, págs. 158-162):

1PL gestiona la logística interna. En general, los representantes de este tipo son pequeñas empresas que proporcionan todos los servicios por sí mismos utilizando sus propios recursos, instalaciones y personal; es decir, utilizan sus propios recursos para gestionar toda la cadena de suministro.

2PL proporciona a las empresas funciones básicas necesarias, como el transporte, el manejo de materiales y el almacenamiento. Por ejemplo, contratar un operador de almacén como subcontratista para la ejecución operativa de una tarea logística y de transporte claramente definida. La organización y el seguimiento siguen siendo responsabilidades del fabricante. La relación con el proveedor solo se basa en los costes y es a corto plazo.

3PL realiza todo el proceso logístico y ofrece variedad de actividades especializadas. Por ejemplo, compañías como DHL Supply Chain o DB Schenker ofrecen una gestión integral de la cadena de suministros desde la recepción de mercancías, tanto locales como importadas, el almacenamiento, la monitorización del stock o la distribución al cliente final, incluida la logística inversa. En estos casos, el cliente contrata al proveedor para que este optimice, con recursos propios o subcontratados, todo el flujo logístico.

Los servicios básicos que ofrece un 3PL son:

- Gestión de transportes o de mercancías (incluida la tecnología, la contabilidad de mercancías y los servicios alrededor de las reclamaciones).

- Almacenamiento.

- Gestión de la distribución.

- Consolidación de mercancías.

4PL es un desarrollo evolutivo del 3PI. La ventaja principal del 4PL es la posibilidad de actuar en nombre del cliente. Por ejemplo, Amazon es un proveedor que, además de ofrecer los servicios de un $3 \mathrm{PL}$, tiene la capacidad de integrar de forma completa el negocio de su cliente en su cadena de suministro. De este modo engloba también la venta de sus productos y la monitorización de los resultados. Servicios que ofrece un $4 \mathrm{PL}^{2}$ :

2. El concepto de 4PL fue introducido hace apenas unos años por Andersen Consulting (1996), en la actualidad Accenture, como un integrador que reúne los recursos, capacidades 
- Monitorización, planificación y optimización de cadenas de suministro.

- Integración de sistemas informáticos.

- Monitorización de pedidos dentro de las cadenas de suministro.

- Optimización del proceso de entrega.

- Evaluación de gestión dentro de las cadenas de suministro.

- Seguimiento de la calidad.

5PL se está desarrollando actualmente en respuesta a la aparición del comercio electrónico. El componente clave de I5PL es la informática y el uso de las TIC y el $\left.\mathrm{B}\right|^{3}$ como valor añadido.

Actualmente, el tipo de proveedores de servicios logísticos más populares son el 3PL y el 4PL. El 4PL gestiona una red de operadores 3PL, y así delega la ejecución de las funciones logísticas en los operadores 3PL que más se adecúen a cada caso. Ello no impide que un operador 3PL, que disponga de grandes infraestructuras, pueda ofrecer servicios de valor añadido aprovechando sus propios activos y evolucionando así hasta competir como operador 4PL.

En la práctica, hay muchos casos en los que las fronteras entre ser 3PL o 4PL son más difusas. Los operadores 3PL han tenido que mantener la competitividad ofreciendo cada vez más servicios. Y, a veces, estos pueden solaparse con lo que un operador 4PL ofrece.

En términos generales, las empresas que subcontratan parte de su logística a un $\mathrm{PPL}$ obtienen potencialmente un ahorro medio de:

- Aumento del $6 \%$ de la tasa de consolidación de pedidos.

- Aumento del $5 \%$ en la precisión de las entregas.

- $9 \%$ en reducciones de costes logísticos.

- $5 \%$ en reducciones de costes de inventario.

- $15 \%$ en reducciones fijas en estructura logística.

Hay que tener en cuenta que, incluso si la externalización proporciona un valor inicial, esto no significa que una organización pueda dejar de prestar atención al rendimiento de su propia logística. Un seguimiento próximo puede ayudar a colaborar con los proveedores para mejorar el rendimiento de forma que se beneficien ambas partes.

y tecnología de su propia organización y otras organizaciones, incluidos los proveedores de 3PL, para diseñar, generar y ejecutar soluciones integrales de la cadena de suministro. 3. $\mathrm{Bl}=$ business inteligence. 
Un operador 4PL tendría que ser independiente, al no disponer de activos físicos que rentabilizar (a diferencia de los 3PL), centrándose en conseguir la máxima eficiencia en la gestión de la cadena de suministro. El operador 4PL manejará una red de operadores 3PL y así podrá delegar la ejecución de las funciones logísticas en aquellos que más se adecúen a cada caso.

\section{Casos de éxito y fracaso en la externalización logística}

Muchas veces, el éxito o el fracaso en el cambio de modelo logístico dependen de la visión de las prioridades y, especialmente, de su integración en el plan de negocio general de la compañía.

La empresa MANGO tenía como prioridad asegurar el surtido de sus colecciones en todas las tiendas y en poco tiempo, sin roturas de stock y gestionando los excedentes, especialmente en la zona de las islas Canarias.

Las necesidades que se generaron para conseguir el objetivo de la compañía fueron las siguientes:

- Externalizar actividad logística de gestión y almacenamiento; de esta manera se especializaba una función con el objetivo de mejorar la productividad, variar costes y tener un mejor control sobre el proceso.

- Almacenamiento del excedente de temporada.

- Gestión y control del excedente.

- Realización de servicios de distribución física entre la totalidad de la red de puntos de venta y outlet de Canarias.

Los resultados conseguidos en este caso fueron los siguientes:

- Reducción del coste logístico vinculado a la gestión de inmovilizado y recursos implícitos de una actividad logística propia en un $15 \%$.

- Almacenamiento en centros logísticos tinerfeños y Las Palmas, según la procedencia de las expediciones, sean de establecimientos de la provincia correspondiente, con el subsiguiente impacto en costes de transporte y plazo de entrega en las tiendas.

- Control de la logística inversa procedente de los puntos de venta de todas las islas.

También tenemos casos en que los pequeños detalles o la gestión, quizás apresurada, comporta un mal resultado derivado de la externalización de los servicios. Si el outsourcing es aplicado de forma errónea, se corre el riesgo de fracasar en el intento y poner incluso en peligro la existencia de la propia compañía. 
Por ejemplo, bajo instrucciones del responsable de la compañía en Estados Unidos, una manufacturera del sector del retail cambió a un modelo de outsourcing de forma completa todas las actividades de logística. La razón que se utilizó para justificar esta decisión fue el cambio de mentalidad de los clientes. Dado que la mayoría de clientes estaban solicitando entregas más pequeñas y frecuentes, pensaron internamente que no tenían suficiente experiencia para implementar una organización just in time y que, si se implementaba de forma errónea, se incrementarían los costes. La decisión de buscar a un tercero como proveedor de este servicio se dio de forma muy rápida, y la compañía tuvo que buscar proveedores, firmar el contrato, transferir la actividad y pasar a un estado operativo en menos de seis meses. Pronto, tras la firma del contrato, las cosas empezaron a salir mal porque el proveedor no cumplió las expectativas. Los pedidos eran entregados con mucho retraso, incluso algunos no se llegaban a entregar nunca, y el inventario caía rápidamente.

El impacto que se generó afectó tanto a los costes logísticos como, sobre todo, a las ventas de producto. Los clientes estaban molestos por el bajo nivel de servicio recibido y empezaron a comprar a otros proveedores.

En este caso, aunque en el contrato firmado inicialmente se hubieran incluido penalizaciones en caso de no cumplir con los servicios contratados, la aplicación de estas penalizaciones no conseguiría nunca compensar el impacto sobre la «supervivencia» de la compañía. Por tanto, es imprescindible tratar la migración de un modelo logístico como un proyecto con todas sus fases de estudio, definición de procesos, plan piloto y seguimiento de resultados. Y, sobre todo, es necesario un PLAN DE CONTINGENCIA que asegure el nivel de servicio esperado por los clientes, sea cual sea nuestra situación interna.

\section{Conclusiones}

La externalización logística es una tendencia mundialmente consolidada que atrae a las empresas por sus numerosas ventajas y posibilidades. Las principales razones del outsourcing son la reducción de costes y riesgos, la posibilidad de centrarse en las actividades de mayor valor añadido, la mejora de la calidad y la mayor eficiencia, flexibilidad y productividad.

Actualmente, las empresas pueden elegir cualquier tipo de proveedor, desde el más sencillo hasta un gran integrador que proporcione todos los servicios necesarios de acuerdo con los requisitos especificados. Los tipos más populares son los $3 \mathrm{PL}$ y $4 \mathrm{PL}$. El proveedor $4 \mathrm{PL}$ actúa como integrador de proveedores de 3PL y, gracias a esto, 4PL y $3 \mathrm{PL}$ no son competidores sino que en muchos casos se complementan. El 3PL puede ser, por ejemplo, propietario de instalaciones de 
transporte y almacenamiento; y el 4PL puede utilizarlas para generar las mejores soluciones para los clientes.

Asimismo, es esencial seleccionar el proveedor adecuado. El proceso de selección incluye la evaluación de la capacidad de respuesta del proveedor, la gestión financiera y de calidad, su reputación y la cuota de mercado. Por último, y después de iniciar una relación con un proveedor, es importante mantener una buena conexión con el socio. Por eso, la comunicación, la confianza y el contrato firmado tienen un papel importante.

\section{Referencias bibliográficas}

BRANCH, A. E. (2009). Global Supply Chain Management and International Logistics. Reino Unido: Routledge.

CHRISTOPHER, M. (2016). Logistics and supply chain management (5. ${ }^{\mathrm{a}}$ ed.). Financial Times/Pearson Education.

CORDON, C.; HALD, K. S.; SEIFERT, R. W. (2012). Strategic supply chain management. Londres / Nueva York: Routledge.

TROACĂ, V. A.; BODISLAV, D. A. (2012) «Outsourcing. The Concept». Theoretical and Aplplied Economics [artículo en línea]. Vol. XIX, n. ${ }^{\circ}$ 6(571), págs. 51-58. $<\mathrm{http}$ ://store.ectap.ro/articole/734.pdf>

LANGLEY, J. et al. (2017). 2017 Trird-Party Logistics Study. The State of Logistics Outsourcing. Capgemini Comsulting.

WATERS, D. (2014). Global logistics. New directions in supply chain management (7. ${ }^{a}$ ed.). Reino Unido.

\section{Webgrafía}

http://www.businessdictionary.com/definition/outsourcing.html http://www.industryweek.com/logistics/outsourcing-does-not-guarantee-superiorwarehouse-performance https://www.chrobinson.com/en-us/resources/case-studies-download/toshibacase-study/ 


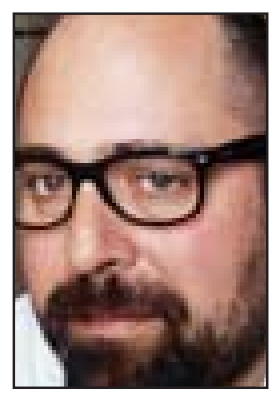

\section{Oriol Glosa Noguera oclosa@uoc.edu Profesor colaborador del máster de Dirección de operaciones y logística integral (UOC)}

EMBA en Negocios Internacionales, máster en Logística Integral y Operaciones, máster universitario en RSC y diplomado en Ciencias Empresariales. En el campo profesional ha estado siempre vinculado a las áreas de logística y compras tanto en el ámbito nacional como internacional. Empezó en Excel Logistics como responsable de planificación Inbound, a continuación se incorporó a Nike como team leader supply chain en la central de Hilversum (Holanda). Ha trabajado como service manager en Akzo Nobel, local supply chain manager y global category manager en Gunnebo. Actualmente trabaja como director de compras en el Grupo Constant. Es profesor/colaborador en la UOC de las siguientes asignaturas: Implantación del modelo lean de negocio, Aprovisionamiento y compras, Filosofía lean de las operaciones, Logística inversa y Gestión de almacenes. Finalmente, colabora en la redacción de artículos en diarios y revistas de ámbito generalista y académico.

Los textos publicados en esta revista están sujetas -salvo que se indique el contrario- a una licencia de Reconocimiento 3.0 España de Creative Commons. Podéis copiarlos, distribuirlos, comunicarlos públicamente y hacer obras derivadas siempre que reconozcáis los créditos de las obras (autoría, nombre de la revista, institución editora) de la manera especificada por los autores o por la revista. La licencia completa se puede consultar en http://creativecommons.org/licenses/by/3.0/es/deed.ca.

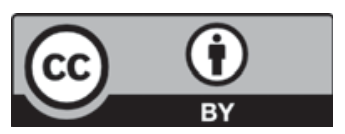

